\title{
Some Characterization Results Based on Conditional Expectation of Function of Dual Generalized Order Statistics
}

\author{
M. I. Khan \\ Department of Statistics \& Operations Research \\ Aligarh Muslim University \\ Aligarh, 202 002. India \\ izhar.stats@gmail.com \\ M. Faizan \\ Department of Statistics \& Operations Research \\ Aligarh Muslim University \\ Aligarh, 202 002. India \\ mdfaizan02@gmail.com
}

\begin{abstract}
Two families of probability distributions are characterized through the conditional expectations of dual generalized order statistics ( $d g o s)$ and spacing of two dgos conditioned on a non-adjacent dual generalized order statistics. Further, some of its deductions are also discussed.
\end{abstract}

Keywords: Characterization, Conditional expectation, Dual generalized order statistics, Probability distributions.

\section{Introduction}

The concept of generalized order statistics ( $g o s$ ) has been introduced as a unified approach to a variety of models of ordered random variables with different interpretation (Kamps, 1995), such as ordinary order statistics, sequential order statistics, progressive type II censoring, record values and Pfeifer's records. Generalized order statistics serve as a common approach to a structural similarities and analogies. Several of these models can be effectively applied, e.g., in reliability theory. Using this concept of gos, Burkschat et al. (2003) introduced the concept of dual generalized order statistics (dgos) that enables a common approach to descending ordered random variables like reverse ordered order statistics, lower record values etc.

Let $X_{1}, X_{2}, \ldots, X_{n}$ be a sequence of independent and identically distributed (iid) random variable with absolutely continuous distribution function $(d f) F(x)$ and the probability density function $(p d f) \quad f(x), x \in(\alpha, \beta)$. Further, let $n \in N, n \geq 2, k \geq 1$, $\tilde{m}=\left(m_{1}, m_{2}, \cdots, m_{n-1}\right) \in \mathfrak{R}^{n-1}, M_{r}=\sum_{j=r}^{n-1} m_{j}$, such that $\gamma_{r}=k+n-r+M_{r}>1$, for all $r \in\{1,2, \ldots, n-1\}$. Then, $X^{\prime}(r, n, \tilde{m}, k), r=1,2, \ldots, n$ are called $d g o s$ if their joint $p d f$ is given by

$$
k\left(\prod_{j=1}^{n-1} \gamma_{j}\right)\left(\prod_{i=1}^{n-1}\left[F\left(x_{i}\right)\right]^{m_{i}} f\left(x_{i}\right)\right)\left[F\left(x_{n}\right)\right]^{k-1} f\left(x_{n}\right)
$$

for $F^{-1}(1) \geq x_{1} \geq x_{2} \geq \ldots \geq x_{n}>F^{-1}(0)$. 


\section{I. Khan, M. Faizan}

Here we will assume two cases:

Case I: $m_{1}=m_{2}=\cdots=m_{n-1}=m$.

The $p d f$ of the $r^{\text {th }} d g o s$ is given by (Burkschat et al., 2003)

$$
f_{X^{\prime}(r, n, m, k)}(x)=\frac{c_{r-1}}{(r-1) !}[F(x)]^{\gamma_{r}-1} f(x)\left[g_{m}(F(x))\right]^{r-1}
$$

The joint $p d f$ of the $r^{\text {th }}$ and $s^{\text {th }} d g o s$ is

$$
\begin{aligned}
& f_{X^{\prime}(r, n, m, k), X^{\prime}(s, n, m, k)}(x, y)=\frac{c_{s-1}}{(r-1) !(s-r-1) !}[F(x)]^{m} f(x) g_{m}^{r-1}(F(x)) \\
& \times\left[h_{m}(F(y))-h_{m}(F(x))\right]^{s-r-1}[F(y)]^{\gamma_{s}-1} f(y), \quad \alpha \leq y<x \leq \beta,
\end{aligned}
$$

where

$$
h_{m}(x)= \begin{cases}-\frac{1}{m+1} x^{m+1} & , m \neq-1 \\ -\log x & , m=-1\end{cases}
$$

and

$$
g_{m}(x)=h_{m}(x)-h_{m}(1), \quad x \in[0,1) .
$$

The conditional $p d f$ of $X^{\prime}(s, n, m, k)$ given $X^{\prime}(r, n, m, k)=x, 1 \leq r<s \leq n$

$$
\begin{aligned}
f_{s \mid r}(y \mid x) & =\frac{c_{s-1}}{(s-r-1) ! c_{r-1}} \\
& \times \frac{\left[(F(x))^{m+1}-(F(y))^{m+1}\right]^{s-r-1}[F(y)]^{\gamma_{s}-1}}{(m+1)^{s-r-1}[F(x)]^{\gamma_{r+1}}} f(y)
\end{aligned}
$$

Case II: $\gamma_{i} \neq \gamma_{j} i \neq j, i, j=1, \ldots, n-1$.

The $p d f$ of the $r^{\text {th }} d g o s$ is given by (Burkschat et al., 2003)

$$
f_{X^{\prime}(r, n, \tilde{m}, k)}(x)=c_{r-1} f(x) \sum_{i=1}^{r} a_{i}(r)[F(x)]^{\gamma_{i}-1}
$$

and the joint $p d f$ of the $r^{\text {th }}$ and $s^{\text {th }} d g o s$ is

$$
\begin{aligned}
f_{X^{\prime}(r, n, \widetilde{m}, k), X^{\prime}(s, n, \widetilde{m}, k)}(x, y)=c_{s-1} & \sum_{j=r+1}^{s} a_{j}^{(r)}(s)\left[\frac{F(y)}{F(x)}\right]^{\gamma_{j}} \\
& \times\left(\sum_{i=1}^{r} a_{i}(r)[F(x)]^{\gamma_{i}}\right) \frac{f(x)}{F(x)} \frac{f(y)}{F(y)}
\end{aligned}
$$

where

$$
a_{i}(r)=\prod_{\substack{j=1 \\ j \neq i}}^{r} \frac{1}{\left(\gamma_{j}-\gamma_{i}\right)}, \quad \gamma_{j} \neq \gamma_{i}, \quad 1 \leq i \leq r \leq n
$$

and

$$
a_{i}^{(r)}(s)=\prod_{\substack{j=r+1 \\ j \neq i}}^{s} \frac{1}{\left(\gamma_{j}-\gamma_{i}\right)}, \quad \gamma_{j} \neq \gamma_{i}, \quad r+1 \leq i \leq s \leq n
$$


Thus, the conditional $p d f$ of $X^{\prime}(s, n, \tilde{m}, k)$ given $X^{\prime}(r, n, \tilde{m}, k)=x, 1 \leq r<s \leq n$ is

$$
f_{s \mid r}(y \mid x)=\frac{c_{s-1}}{c_{r-1}} \sum_{i=r+1}^{s} a_{i}^{(r)}(s)\left(\frac{F(y)}{F(x)}\right)^{\gamma_{i}-1} \frac{f(y)}{F(x)}, \quad x>y
$$

If $m=0, k=1$, then $X^{\prime}(r, n, m, k)$ reduces to $X_{n-r+1: n}$, the $(n-r+1)^{\text {th }}$ order statistics, from a sample of size $n$ and when $m=-1$, then $X^{\prime}(r, n, m, k)$ reduces to the $r^{\text {th }} k-$ lower record value (Pawlas and Szynal, 2001). A number of results on characterization of distributions of dual generalized order statistics are available in the literature. For a detailed survey one may refer to Ahsanullah (2004), Mbah and Ahsanullah (2007), Khan et al. (2009) and Khan et al. (2010) and references contained therein. In this paper, two general classes of distributions

$$
F(x)=e^{-a h(x)}, a \neq 0, x \in(\alpha, \beta)
$$

and

$$
F(x)=[a h(x)+b]^{c}, x \in(\alpha, \beta)
$$

have been characterized through conditional expectation of function of dgos, where $a, b, c$ and $h(x)$ are so chosen that $F(x)$ in (1.10) and (1.11) are $d f$.

It may be noted that the $d f F(x)$ and the $p d f f(x)$ in (1.10) and (1.11) are related respectively as

$$
F(x)=-\frac{f(x)}{a h^{\prime}(x)}
$$

and

$$
\frac{f(x)}{F(x)}=\frac{a c h^{\prime}(x)}{[a h(x)+b]}
$$

Khan et al. (2010) have characterized the general class of distributions through conditional expectation of dgos conditioned on non-adjacent dgos. We have extended the result of Khan et al. (2010) for the difference of the conditional expectations conditioned on non-adjacent $d g o s$, its particular cases for order statistics, lower record statistics as obtained by Khan et al. (2011) and Faizan and Khan (2011) are discussed.

\section{Characterizations of distributions when}

$$
\gamma_{i} \neq \gamma_{j} i \neq j, i, j=1, \ldots, n-1 \text {. }
$$

Theorem 2.1: Let $X$ be an absolutely continuous random variable with the $d f F(x)$ and the $p d f f(x)$ in the interval $(\alpha, \beta)$, where $\alpha$ and $\beta$ may be finite or infinite, then for $1 \leq r<s<t \leq n$,

$$
\begin{aligned}
E\left[h\left\{X^{\prime}(s, n, \tilde{m}, k)\right\}-h\left\{X^{\prime}(t, n, \tilde{m}, k)\right\} \mid X^{\prime}\right. & (l, n, \tilde{m}, k)=x] \\
& =-\frac{1}{a} \sum_{j=t+1}^{s} \frac{1}{\gamma_{j}}, l=r, r+1
\end{aligned}
$$




\section{I. Khan, M. Faizan}

if and only if

$$
F(x)=e^{-a h(x)}, a \neq 0
$$

where $h(x)$ is a monotonic and differentiable function of $x$ such that $h(x) \rightarrow 0$ as $x \rightarrow \beta$ and $h(x) F(x) \rightarrow 0$ as $x \rightarrow \alpha$.

Proof: First we shall prove that (2.2) implies (2.1). It can be seen (Athar et al., 2008) that

$$
\begin{aligned}
E\left[h\left\{X^{\prime}(t, n, \tilde{m}, k)\right\}-h\left\{X^{\prime}(\right.\right. & \left.t-1, n, \tilde{m}, k)\} \mid X^{\prime}(r, n, \tilde{m}, k)=x\right] \\
= & \frac{c_{t-2}}{c_{r-1}} \sum_{i=r+1}^{t} a_{i}^{(r)}(t) \int_{\alpha}^{x} h^{\prime}(y)\left(\frac{F(y)}{F(x)}\right)^{\gamma_{i}} d y
\end{aligned}
$$

Therefore, for $1 \leq r<s<t \leq n$

$$
\begin{aligned}
E\left[h\left\{X^{\prime}(s, n, \tilde{m}, k)\right\}-h\left\{X^{\prime}(t, n, \tilde{m}, k)\right\} \mid X^{\prime}(r, n, \tilde{m}, k)=x\right] \\
=\sum_{i=0}^{s-t-1} E\left[h\left\{X^{\prime}(s-i, n, \tilde{m}, k)\right\}-h\left\{X^{\prime}(s-i-1, n, \tilde{m}, k)\right\} \mid X^{\prime}(r, n, \tilde{m}, k)=x\right] \\
=\sum_{j=t+1}^{s} E\left[h\left\{X^{\prime}(j, n, m, k)\right\}-h\left\{X^{\prime}(j-1, n, m, k)\right\} \mid X^{\prime}(r, n, m, k)=x\right] \\
=\sum_{j=t+1}^{s} \frac{c_{j-2}}{c_{r-1}} \sum_{i=r+1}^{j} a_{i}^{(r)}(j) \int_{\alpha}^{x} h^{\prime}(y)\left(\frac{F(y)}{F(x)}\right)^{\gamma_{i}} d y \\
=-\frac{1}{a} \sum_{j=t+1}^{s} \frac{1}{\gamma_{j}}, \text { in view of (1.9) and (1.12) }
\end{aligned}
$$

This proves the necessary part.

For the sufficiency part, we have at $c=\frac{1}{a} \sum_{j=t+1}^{s} \frac{1}{\gamma_{j}}$

$$
\begin{aligned}
& E\left[h\left\{X^{\prime}(t, n, \tilde{m}, k)\right\}-\right.\left.h\left\{X^{\prime}(s, n, \tilde{m}, k)\right\} \mid X^{\prime}(r, n, \tilde{m}, k)=x\right]=c \\
& \frac{c_{t-1}}{c_{r-1}} \sum_{i=r+1}^{t} a_{i}^{(r)}(t) \int_{\alpha}^{x} h(y)\left(\frac{F(y)}{F(x)}\right)^{\gamma_{i}} \frac{f(y)}{F(y)} d y \\
& \quad-\frac{c_{s-1}}{c_{r-1}} \sum_{i=r+1}^{s} a_{i}^{(r)}(s) \int_{\alpha}^{x} h(y)\left(\frac{F(y)}{F(x)}\right)^{\gamma_{i}} \frac{f(y)}{F(y)} d y=c
\end{aligned}
$$

Differentiating (2.3) w.r.t. $x$ we have

$$
\begin{aligned}
\frac{f(x)}{F(x)} \frac{c_{t-1}}{c_{r-1}} \sum_{i=r+1}^{t} a_{i}^{(r)}(t) h(x)-\frac{f(x)}{F(x)} \frac{c_{t-1}}{c_{r-1}} \sum_{i=r+1}^{t} \gamma_{i} a_{i}^{(r)}(t) \\
\times \int_{\alpha}^{x} \frac{h(y)[F(y)]^{\gamma_{i}-1} f(y)}{[F(x)]^{\gamma_{i}}} d y-\frac{f(x)}{F(x)} \frac{c_{s-1}}{c_{r-1}} \sum_{i=r+1}^{s} a_{i}^{(r)}(s) h(x) \\
+\frac{f(x)}{F(x)} \frac{c_{s-1}}{c_{r-1}} \sum_{i=r+1}^{s} \gamma_{i} a_{i}^{(r)}(s) \int_{\alpha}^{x} \frac{h(y)[F(y)]^{\gamma_{i}-1} f(y)}{[F(x)]^{\gamma_{i}}} d y=0
\end{aligned}
$$


Rearranging the terms in (2.4) and noting that $\sum_{i=r+1}^{s} a_{i}^{(r)}(s)=0, c_{r}=\gamma_{r+1} c_{r-1}$ and $a_{i}^{(r+1)}(t)=\left(\gamma_{r+1}-\gamma_{i}\right) a_{i}^{(r)}(t)$, we get

$$
\left[g_{t \mid r}(x)-g_{t \mid r+1}(x)\right]-\left[g_{s \mid r}(x)-g_{s \mid r+1}(x)\right]=0
$$

where

$$
g_{s \mid r}(x)=E\left[h\left\{X^{\prime}(s, n, \tilde{m}, k)\right\} \mid X^{\prime}(r, n, \tilde{m}, k)=x\right]
$$

or,

$$
g_{t \mid r}(x)-g_{s \mid r}(x)=g_{t \mid r+1}(x)-g_{s \mid r+1}(x)=\cdots=g_{t \mid s}(x)-g_{s \mid s}(x)=c
$$

Noting that $g_{s \mid s}(x)=h(x)$, we have

$$
g_{t \mid s}(x)=h(x)+c
$$

i.e. $E\left[h\left\{X^{\prime}(t, n, \tilde{m}, k)\right\} \mid X^{\prime}(s, n, \tilde{m}, k)=x\right]=h(x)+\frac{1}{a} \sum_{j=s+1}^{t} \frac{1}{\gamma_{j}}$

Using the result (Khan et al., 2006)

$$
E\left[h\left\{X^{\prime}(t, n, \tilde{m}, k)\right\} \mid X^{\prime}(s, n, \widetilde{m}, k)=x\right]=g_{t \mid s}(x),
$$

implies

$$
F(x)=e^{-\int_{x}^{\beta} A(u) d u}
$$

where

$$
A(u)=\frac{g_{t \mid s}^{\prime}(u)}{\gamma_{s+1}\left[g_{t \mid s+1}(u)-g_{t \mid s}(u)\right]}=-a h^{\prime}(u)
$$

we get,

$$
F(x)=e^{-a h(x)}, a \neq 0
$$

and hence the Theorem.

Remark 2.1: At $s=r, E\left[h\left\{X^{\prime}(t, n, \tilde{m}, k)\right\} \mid X^{\prime}(s, n, \tilde{m}, k)=x\right]=\frac{1}{a} \sum_{j=s+1}^{t} \frac{1}{\gamma_{j}}+h(x)$ as obtained by Khan et al. (2010).

Remark 2.2: At $m=0, k=1$, we will get the following result for order statistics for $1 \leq r<s<t \leq n$

$$
\begin{aligned}
& E\left[h\left\{\left(X_{s: n}^{\prime}\right)\right\}-h\left\{\left(X_{t: n}^{\prime}\right)\right\} \mid X_{r: n}^{\prime}=x\right]=-\frac{1}{a} \sum_{j=t+1}^{s} \frac{1}{n-j+1} \\
& E\left[h\left\{\left(X_{s: n}\right)\right\}-h\left\{\left(X_{r: n}\right)\right\} \mid X_{t: n}=y\right]=-\frac{1}{a} \sum_{j=r}^{s-1} \frac{1}{j}
\end{aligned}
$$

as obtained by Khan et al. (2011).

Remark 2.3: At $m=-1, k=1$ and $c=\frac{1}{a}$ it will give result for lower record statistics as obtained by Faizan and Khan (2011). 
Corollary 2.1: Under the conditions as given in Theorem 2.1 and for $1 \leq r<s<t \leq n$

$$
\begin{array}{r}
E\left[h\left\{X^{\prime}(s, n, \tilde{m}, k)\right\}-h\left\{X^{\prime}(r, n, \tilde{m}, k)\right\}\right]+h(x) \\
=E\left[h\left\{X^{\prime}(s, n, \tilde{m}, k)\right\} \mid X^{\prime}(r, n, \widetilde{m}, k)=x\right]
\end{array}
$$

if and only if

$$
F(x)=e^{-a h(x)}, a \neq 0
$$

Proof: The proof from Theorem 2.1 and Remark 2.1.

Further, putting $m=0, k=1$ in equation (2.9), we will get the result for order statistics as follows,

$$
E\left[h\left\{\left(X_{r: n}\right)\right\}-h\left\{\left(X_{s: n}\right)\right\}\right]+h(x)=E\left[h\left\{\left(X_{r: n}\right)\right\} \mid X_{s: n}=x\right]
$$

Theorem 2.2: Let $X$ be an absolutely continuous random variable with the $d f F(x)$ and the $p d f f(x)$ in the interval $(\alpha, \beta)$, where $\alpha$ and $\beta$ may be finite or infinite, then for $1 \leq r<s<t \leq n$,

$$
\begin{aligned}
& E\left[h\left\{X^{\prime}(t, n, \tilde{m}, k)\right\} \mid X^{\prime}(l, n, \tilde{m}, k)=x\right] \\
= & a_{t \mid s}^{*} E\left[h\left\{X^{\prime}(s, n, \tilde{m}, k)\right\} \mid X^{\prime}(l, n, \tilde{m}, k)=x\right]+b_{t \mid s}^{*} \quad, l=r, r+1
\end{aligned}
$$

if and only if

$$
F(x)=[a h(x)+b]^{c}
$$

where

$$
a_{t \mid s}^{*}=\prod_{j=s+1}^{t} \frac{c \gamma_{j}}{1+c \gamma_{j}} \text { and } b_{t \mid s}^{*}=-\frac{b}{a}\left(1-a_{t \mid s}^{*}\right)
$$

Proof: First we shall prove that (2.12) implies (2.11). In view of Khan et al. (2010), we have

$$
\begin{gathered}
E\left[h\left\{X^{\prime}(t, n, \tilde{m}, k)\right\} \mid X^{\prime}(r, n, \tilde{m}, k)=x\right]=a_{t \mid r}^{*} h(x)+b_{t \mid r}^{*} \\
=a_{t \mid r}^{*}\left(h(x)+\frac{b}{a}\right)-\frac{b}{a}
\end{gathered}
$$

where

$$
a_{t \mid r}^{*}=\prod_{j=r+1}^{t} \frac{c \gamma_{j}}{1+c \gamma_{j}}=a_{s \mid r}^{*} a_{t \mid s}^{*}
$$

and

$$
\begin{gathered}
b_{t \mid r}^{*}=-\frac{b}{a}\left(1-a_{t \mid r}^{*}\right) \\
\begin{aligned}
E\left[h\left\{X^{\prime}(t, n, \tilde{m}, k)\right\} \mid X^{\prime}(r, n, \tilde{m}, k)=x\right]=a_{t \mid s}^{*} a_{s \mid r}^{*}\left(h(x)+\frac{b}{a}\right)-\frac{b}{a} \\
=a_{t \mid s}^{*} a_{s \mid r}^{*}\left[\left(h(x)+\frac{b}{a}\right)-\frac{b}{a}\right]+\frac{b}{a} a_{t \mid s}^{*}-\frac{b}{a} \\
=a_{t \mid s}^{*} E\left[h\left\{X^{\prime}(s, n, \tilde{m}, k)\right\} \mid X^{\prime}(r, n, \tilde{m}, k)=x\right]+b_{t \mid s}^{*}
\end{aligned}
\end{gathered}
$$

This proves the necessary part. 
For the sufficiency part, we have

$$
\begin{aligned}
\frac{c_{t-1}}{c_{r-1}} \sum_{i=r+1}^{t} a_{i}^{(r)}(t) \int_{\alpha}^{x} h(y)\left[\frac{F(y)}{F(x)}\right]^{\gamma_{i}} \frac{f(y)}{F(y)} d y & \\
= & a_{t \mid s}^{*} \frac{c_{s-1}}{c_{r-1}} \sum_{i=r+1}^{s} a_{i}^{(r)}(s) \int_{\alpha}^{x} h(y)\left[\frac{F(y)}{F(x)}\right]^{\gamma_{i}} \frac{f(y)}{F(y)} d y+b_{t \mid s}^{*}
\end{aligned}
$$

Differentiating (2.14) w.r.t. $x$ and rearranging, we get

$$
\begin{aligned}
& {\left[\frac{c_{t-1}}{c_{r-1}} \sum_{i=r+1}^{t} a_{i}^{(r)}(t) h(x)-\frac{c_{t-1}}{c_{r-1}} \sum_{i=r+1}^{t} \gamma_{i} a_{i}^{(r)}(t)\right.}\left.\int_{\alpha}^{x} \frac{h(y)[F(y)]^{\gamma_{i}-1} f(y)}{[F(x)]^{\gamma_{i}}} d y\right] \\
&=a_{t \mid s}^{*}\left[\frac{c_{s-1}}{c_{r-1}} \sum_{i=r+1}^{s} a_{i}^{(r)}(s) h(x)-\frac{c_{s-1}}{c_{r-1}} \sum_{i=r+1}^{s} \gamma_{i} a_{i}^{(r)}(s)\right. \\
&\left.\times \int_{\alpha}^{x} \frac{h(y)[F(y)]^{\gamma_{i}-1} f(y)}{[F(x)]^{\gamma_{i}}} d y\right]
\end{aligned}
$$

After noting that $\sum_{i=r+1}^{s} a_{i}^{(r)}(s)=0, c_{r}=\gamma_{r+1} c_{r-1}$ and $a_{i}^{(r+1)}(t)=\left(\gamma_{r+1}-\gamma_{i}\right) a_{i}^{(r)}(t)$, we get

$$
\begin{aligned}
& \frac{\gamma_{r+1} c_{t-1}}{c_{r-1}} \sum_{i=r+1}^{t} a_{i}^{(r)}(t) \int_{\alpha}^{x} \frac{h(y)[F(y)]^{\gamma_{i}-1} f(y)}{[F(x)]^{\gamma_{i}}} d y \\
& -\frac{\gamma_{r+1} c_{t-1}}{c_{r}} \sum_{i=r+2}^{t} a_{i}^{(r+1)}(t) \int_{\alpha}^{x} \frac{h(y)[F(y)]^{\gamma_{i}-1} f(y)}{[F(x)]^{\gamma_{i}}} d y \\
& =a_{t \mid s}^{*}\left[\frac{\gamma_{r+1} c_{s-1}}{c_{r-1}} \sum_{i=r+1}^{s} a_{i}^{(r)}(s) \int_{\alpha}^{x} \frac{h(y)[F(y)]^{\gamma_{i}-1} f(y)}{[F(x)]^{\gamma_{i}}} d y\right. \\
& \left.\quad-\frac{\gamma_{r+1} c_{s-1}}{c_{r}} \sum_{i=r+2}^{s} a_{i}^{(r+1)}(s) \int_{\alpha}^{x} \frac{h(y)[F(y)]^{\gamma_{i}-1} f(y)}{[F(x)]^{\gamma_{i}}} d y\right]
\end{aligned}
$$

That is,

$$
\gamma_{r+1}\left[g_{t \mid r}(x)-g_{t \mid r+1}(x)\right] \quad=a_{t \mid s}^{*} \gamma_{r+1}\left[g_{s \mid r}(x)-g_{s \mid r+1}(x)\right]
$$

where

$$
g_{s \mid r}(x)=E\left[h\left\{X^{\prime}(s, n, \tilde{m}, k)\right\} \mid X^{\prime}(r, n, \tilde{m}, k)=x\right]
$$

or,

$$
\begin{aligned}
g_{t \mid r}(x)-a_{t \mid s}^{*} g_{s \mid r}(x)=g_{t \mid r+1}(x)-a_{t \mid s}^{*} g_{s \mid r+1}(x) & \\
=\cdots & =g_{t \mid s}(x)-a_{t \mid s}^{*} g_{s \mid s}(x)=b_{t \mid s}^{*}
\end{aligned}
$$

Noting that $g_{s \mid s}(x)=h(x)$, we have

$$
g_{t \mid s}(x)=a_{t \mid s}^{*} h(x)+b_{t \mid s}^{*}
$$

i.e.

$$
E\left[h\left\{X^{\prime}(t, n, \tilde{m}, k)\right\} \mid X^{\prime}(s, n, \tilde{m}, k)=x\right]=a_{t \mid s}^{*} h(x)+b_{t \mid s}^{*}
$$


Using the result (Khan et al., 2006)

$$
E\left[h\left\{X^{\prime}(t, n, \tilde{m}, k)\right\} \mid X^{\prime}(s, n, \tilde{m}, k)=x\right]=g_{t \mid s}(x),
$$

implies

$$
F(x)=e^{-\int_{x}^{\beta} A(u) d u}
$$

We get

$$
F(x)=[a h(x)+b]^{c}
$$

and hence the Theorem.

Remark 2.4: It may be seen that when $\gamma_{i} \neq \gamma_{j}$ but $m_{i}=m_{j}=m$, then

$$
\begin{aligned}
& a_{i}^{(r)}(t)=\frac{1}{(m+1)^{t-r-1}}(-1)^{t-i} \frac{1}{(i-r-1) !(t-i) !} \\
& a_{i}(r)=\frac{1}{(m+1)^{r-1}}(-1)^{r-i} \frac{1}{(i-1) !(r-i) !}
\end{aligned}
$$

and consequently (1.5) will reduce to (1.2), (1.6) to (1.3).

Remark 2.5: At $m=0, k=1$, we will get result for order statistics as follows,

$$
\begin{aligned}
E\left[h\left\{\left(X_{t: n}^{\prime}\right)\right\} \mid\right. & \left.\left(X_{r: n}^{\prime}\right)=x\right] \\
& =a_{t \mid s}^{*} E\left[h\left\{\left(X_{s: n}^{\prime}\right)\right\} \mid\left(X_{r: n}^{\prime}\right)=x\right]+b_{t \mid s}^{*}
\end{aligned}
$$

or

$$
E\left[h\left\{\left(X_{r: n}\right)\right\} \mid\left(X_{t: n}\right)=y\right]=a_{r \mid s}^{*} E\left[h\left\{\left(X_{s: n}\right)\right\} \mid\left(X_{t: n}\right)=y\right]+b_{r \mid s}^{*}
$$

where

$$
a_{r \mid s}^{*}=\prod_{j=r}^{s-1} \frac{c j}{1+c j} \text { and } b_{r \mid s}^{*}=-\frac{b}{a}\left(1-a_{r \mid s}^{*}\right)
$$

as obtained by Khan et al.(2011).

Remark 2.6: At $s=r$, it reduces to as obtained by Khan et al. (2010).

Remark 2.7: At $a=-\frac{a}{c}, b=1$ and $c \rightarrow \infty$ then $F(x)=[a h(x)+b]^{c} \rightarrow e^{-a h(x)}$ as obtained in Theorem 2.1 .

\section{Conclusion}

In this paper, conditional expectation of the difference of two dgos conditioned on nonadjacent dgos are considered to characterize the $d f \quad F(x)=e^{-a h(x)}$ whose particular cases are given in the Table 2.1 with proper choice of $a, b, c$ and $h(x)$. Also $F(x)=[a h(x)+b]^{c}$ is characterized through the conditional expectation of $d g o s$ conditioned on non-adjacent $d g o s$ as given in Table 2.2. Further, its various deductions are discussed. 
Table 2.1: Examples based on the distribution function $F(x)=e^{-a h(x)}$

\begin{tabular}{|c|c|c|c|}
\hline Distribution & $F(x)$ & $a$ & $h(x)$ \\
\hline Inverse Weibull & $\begin{array}{c}e^{-\theta x^{-p}} \\
0<x<\infty\end{array}$ & $\theta$ & $x^{-p}$ \\
\hline Power function & $\begin{array}{c}\left(\frac{x}{a}\right)^{p} \\
0<x<a\end{array}$ & $-p$ & $\log (x / a)$ \\
\hline Logistic & $\begin{array}{l}\left(1+e^{-x}\right)^{-1} \\
-\infty<x<\infty\end{array}$ & 1 & $\log \left(1+e^{-x}\right)$ \\
\hline Burr Type II & $\begin{array}{l}\left(1+e^{-x}\right)^{-k} \\
-\infty<x<\infty\end{array}$ & $k$ & $\log \left(1+e^{-x}\right)$ \\
\hline Burr Type III & $\begin{array}{l}\left(1+x^{-c}\right)^{-k} \\
0<x<\infty\end{array}$ & $k$ & $\log \left(1+x^{-c}\right)$ \\
\hline Burr Type IV & $\begin{array}{c}{\left[1+\left(\frac{c-x}{x}\right)^{1 / c}\right]^{-k}} \\
0<x<c\end{array}$ & $k$ & $\log \left[1+\left(\frac{c-x}{x}\right)^{1 / c}\right]$ \\
\hline Burr Type V & $\begin{array}{c}\left(1+c e^{-\tan x}\right)^{-k} \\
-\frac{\pi}{2}<x<\frac{\pi}{2}\end{array}$ & $k$ & $\log \left(1+c e^{-\tan x}\right)$ \\
\hline Burr Type VI & $\begin{array}{r}\left(1+c e^{-k \sinh x}\right)^{-k} \\
-\infty<x<\infty\end{array}$ & $k$ & $\log \left(1+c e^{-k \sinh x}\right)$ \\
\hline Burr Type VII & $\begin{array}{l}\left(\frac{1+\tanh x}{2}\right)^{k} \\
-\infty<x<\infty\end{array}$ & $-k$ & $\log \left(\frac{1+\tanh x}{2}\right)$ \\
\hline Burr Type VIII & $\begin{array}{l}\left(\frac{2}{\pi} \tan ^{-1} e^{x}\right)^{k} \\
-\infty<x<\infty\end{array}$ & $-k$ & $\log \left(\frac{2}{\pi} \tan ^{-1} e^{x}\right)$ \\
\hline Burr Type X & $\begin{array}{c}\left(1-e^{-x^{2}}\right)^{k} \\
0<x<\infty\end{array}$ & $-k$ & $\log \left(1-e^{-x^{2}}\right)$ \\
\hline Burr Type XI & $\begin{array}{c}\left(x-\frac{1}{2 \pi} \sin 2 \pi x\right)^{k} \\
0<x<1\end{array}$ & $-k$ & $\log \left(x-\frac{1}{2 \pi} \sin 2 \pi x\right)$ \\
\hline Gumbel & $\begin{array}{c}\exp \left[-e^{-x}\right] \\
-\infty<x<\infty\end{array}$ & 1 & $e^{-x}$ \\
\hline Extreme value II & $\begin{array}{l}e^{-\left(\frac{\theta}{x}\right)^{p}} \\
0<x<\infty\end{array}$ & $\theta^{p}$ & $x^{-p}$ \\
\hline
\end{tabular}


Table 2.2: Examples based on the distribution function $F(x)=[a h(x)+b]^{c}$

\begin{tabular}{|l|c|c|c|c|c|}
\hline Distribution & $\boldsymbol{F ( x )}$ & $\boldsymbol{a}$ & $\boldsymbol{b}$ & $\boldsymbol{c}$ & $\boldsymbol{h}(\boldsymbol{x})$ \\
\hline Power function & $\begin{array}{c}a^{-p} x^{p} \\
0<x \leq a\end{array}$ & $a^{-q}$ & 0 & $p / q$ & $x^{q}, q \neq 0$ \\
\hline Pareto & $\begin{array}{c}1-a^{p} x^{-p} \\
a \leq x<\infty\end{array}$ & $a^{p}$ & $1-a^{p}$ & 1 & $1-x^{-p}$ \\
\hline Inverse Weibull & $e^{-\theta x^{-p}}$ & 1 & 1 & $x^{-p}$ \\
& $0 \leq x<\infty$ & 1 & 0 & $\theta / q$ & $x^{-p}$ \\
\hline Burr type III & $\left(1+x^{-c}\right)^{-k}$ & 1 & 1 & $-k$ & $e^{-q x^{-p}}, q \neq 0$ \\
\hline Cauchy & $0 \leq x<\infty$ & 1 & 0 & $-k / q$ & $\left(1+\boldsymbol{x}^{-c}\right)^{q}$, \\
& $\frac{1}{2}+\frac{1}{\pi} \tan ^{-1} x$ & $\frac{1}{\pi}$ & $\frac{1}{2}$ & 1 & $\tan ^{-1} x$ \\
\hline
\end{tabular}

\section{Acknowledgement}

The authors are grateful to Professor A.H. Khan, Department of Statistics and Operations Research, Aligarh Muslim University for his valuable suggestions in preparing this paper. We are also thankful to the referees for their useful suggestions, which lead to the improvement of the manuscript.

\section{References}

1. Ahsanullah, M. (2004). A characterization of the uniform distribution by dual generalized order statistics. Comm. Statist. Theory Methods, 33, 2921-2928.

2. Athar Haseeb, Anwar, Z. and Khan, R.U. (2008). On recurrence relations for the expectations of function of lower generalized order statistics. Pakistan. J. Statist., 24, 111-122.

3. Burkschat, M., Cramer, E. and Kamps, U. (2003). Dual generalized order statistics. Metron, LXI (1), 13-26.

4. Faizan, M. and Khan, M.I. (2011). A characterization of continuous distributions through lower record statistics. Prob Stat Forum., 4, 39-43.

5. Kamps, U. (1995). A Concept of Generalized Order Statistics, B.G. Teubner Stuttgart.

6. Khan, A.H., Anwar, Z. and Chishti, S. (2010). Characterization of continuous distributions through conditional expectation of functions of dual generalized order statistics. Pakistan J. Statist., 26, 615-628.

7. Khan, A.H., Khan, R.U. and Yaqub, M. (2006). Characterization of continuous distributions through conditional expectation of functions of generalized order statistics. J. Appl. Probab. Statist., 1, 115-131. 
8. Khan, A.H., Faizan, M. and Haque, Z. (2011). Characterization of continuous distributions via conditional expectations of non-adjacent order statistics. Submitted for publication.

9. Khan, M.J.S., Haque, Z. and Faizan, M. (2009). On characterization of continuous distributions conditioned on a pair of non-adjacent dual generalized order statistics. Aligarh J. Statist., 29,107-119.

10. Mbah, A.K. and. Ahsanullah, M. (2007). Some characterization of the power function distribution based on lower generalized order statistics. Pakistan J. Statist., 23, 139-146.

11. Pawlas, P. and Szynal, D. (2001). Recurrence relations for single and product moments of lower generalized order statistics from the inverse Weibull distributions. Demonstratio Mathematica, XXXIV (2), 353-358. 